Ann. Abeille, 1966, 9 (3), 251-263.

REVUE

\title{
L'INSÉMINATION ARTIFICIELLE DES REINES D'ABEILLES
}

\author{
J. FRESNAYE \\ Station expérimentale d'Apiculture, \\ Centre de Recherches agronomiques du Sud-Est, 84-Montfavet
}

SOMMAIRE

L'auteur fait une revue succincte des travaux les plus marquants concernant les progrès récents de l'insémination artificielle instrumentale des reines d'Abeilles, ainsi qu'une description détaillée de la méthode de travail.

\section{INTRODUCTION}

Nous ne ferons pas un historique détaillé des tentatives d'insémination artificielle des reines d'Abeilles. Depuis RÉAUmur en I740 qui essaya de faire féconder des reines dans des flacons de verre, puis HUBER en I8I4 qui tenta de transporter du sperme dans les voies génitales des reines à l'aide d'un pinceau, de nombreux chercheurs se sont intéressés à ce problème complexe.

En effet, dans les conditions naturelles, les reines vierges ne peuvent être fécondées qu'au cours des vols de fécondation, à grande hauteur et souvent à de grandes distances de leur ruche d'origine. Les essais de fécondation dans des enceintes de toutes tailles, depuis la bouteille de verre jusqu'aux serres de plusieurs centaines de mètre cubes, furent presque toujours négatifs. Cependant, SMiтн (I96I) obtint une fécondation dans une chambre de Renner, mais ce cas isolé ne put être reproduit.

Des essais d'introduction manuelle des parties génitales des mâles dans celles des reines furent également tentés. ChaUvin (I950) présente ces techniques, utilisées notamment en Russie et par LAIDLAW (I932). Il décrit également une méthode dans laquelle un appareil simple doit permettre de faciliter l'introduction du sperme dans les voies génitales de la reine. Mais Triasko (I958), au cours d'expériences 
réunissant $2 \mathrm{I} 4$ reines inséminées manuellement, trouve que $20 \mathrm{p}$. Ioo de celles-ci n'ont pas reçu de sperme et que les meilleures n'en recèlent que $2,2 \mathrm{p}$. Ioo du volume normal. Dans une autre expérience réunissant 75 reines, celles-ci ne donnèrent que du couvain de mâles ou pas du tout de couvain.

C'est surtout dans le domaine de l'insémination artificielle pratiquée à l'aide d'un appareillage spécialement étudié que l'on a enregistré les progrès les plus importants. On peut considérer que l'insémination artificielle instrumentale moderne commence avec les travaux de WATSON (I927). Il préconise l'utilisation d'une microseringue pour le prélèvement et l'injection du sperme et d'un système de contention pour la reine, le tout disposé sous une loupe binoculaire. NoLAN (I932-I937) puis Mackensen et Roberts (I948) construisirent un appareil qui fut utilisé dans le monde entier et qui réunit encore de nombreux partisans. RUTTNER (I964) a modifié cet appareil et propose un montage en plexiglas qui améliore les conditions d'anesthésie et de contention de la reine. L'appareil conçu par LAIDLAW (I949-I953) permet une plus grande précision des divers mouvements, grâce à des vis micrométriques, et réduit les risques de blesser les reines. Les seringues de WATson, NoLAN, LAIDLAW ont été remplacées par celle de MAckeNSEN (I948) qui est actuellement la plus utilisée; récemment VESELY (I96I) a réalisé une pointe de seringue très fine qui améliore la précision de l'opération.

Un progrès important a été réalisé par LAIDLAW (I944) qui a reconnu l'importance du repli vaginal dans l'insémination artificielle. Il est indispensable que ce repli soit écarté, à l'aide d'une sonde spécialement conçue à cet effet, pour permettre le passage de la seringue afin d'obtenir une fécondation correcte.

L'importance capitale de l'anesthésie a été découverte par Mackenser en I947. Jusqu'alors les reines, qui étaient anesthésiées à l'éther ou inséminées sans anesthésie, ne commencaient à pondre que 30 à 50 jours après 1 'insémination. En utilisant le gaz carbonique $\left(\mathrm{CO}_{2}\right)$ comme anesthésique MACKENSEN ramène le délai avant le début de la ponte au voisinage de la normale; l'écart moyen entre reines fécondées naturellement et artificiellement tombe à $I, 9$ jours. L'anesthésie des reines vierges au gaz carbonique permet par ailleurs d'obtenir facilement et rapidement des reines à ponte parthénogénétique, qui ne pondent que des œufs de mâles. Elles sont utilisées en sélection et en génétique de l'abeille.

\section{L'APPAREIL POUR L'INSÉMINATION AR'TIFICIELLE}

L'appareil pour l'insémination artificielle des reines de LAIDLAW (fig. I et 2) est celui que nous avons adopté comme étant le plus perfectionné et le plus pratique. Il se compose de deux parties distinctes, non solidaires, l'appareil de contention de la reine d'une part, le support de la seringue d'autre part. L'appareil de contention de la reine a pour base un socle en acier qui assure une bonne stabilité de l'appareil. Une rotule permettant l'orientation désirée relie ce socle à la chambre d'anesthésie de la reine. Cette chambre est composée d'un étau dont la fermeture est assurée par une vis micrométrique et dont les mâchoires sont garnies de mousse plastique très souple afin de ne pas blesser la reine. Un tube débouche dans la chambre et permet l'arrivée du gaz carbonique qui sert d'anesthésique. De part et d'autre de la chambre d'anesthésie se trouvent deux supports montés sur des glissières horizontales et commandés par des vis micrométriques qui assurent le déplacement vertical du crochet ventral et du crochet dorsal qui peuvent ainsi être avancés ou reculés, montés ou descendus, avec la plus grande précision. 


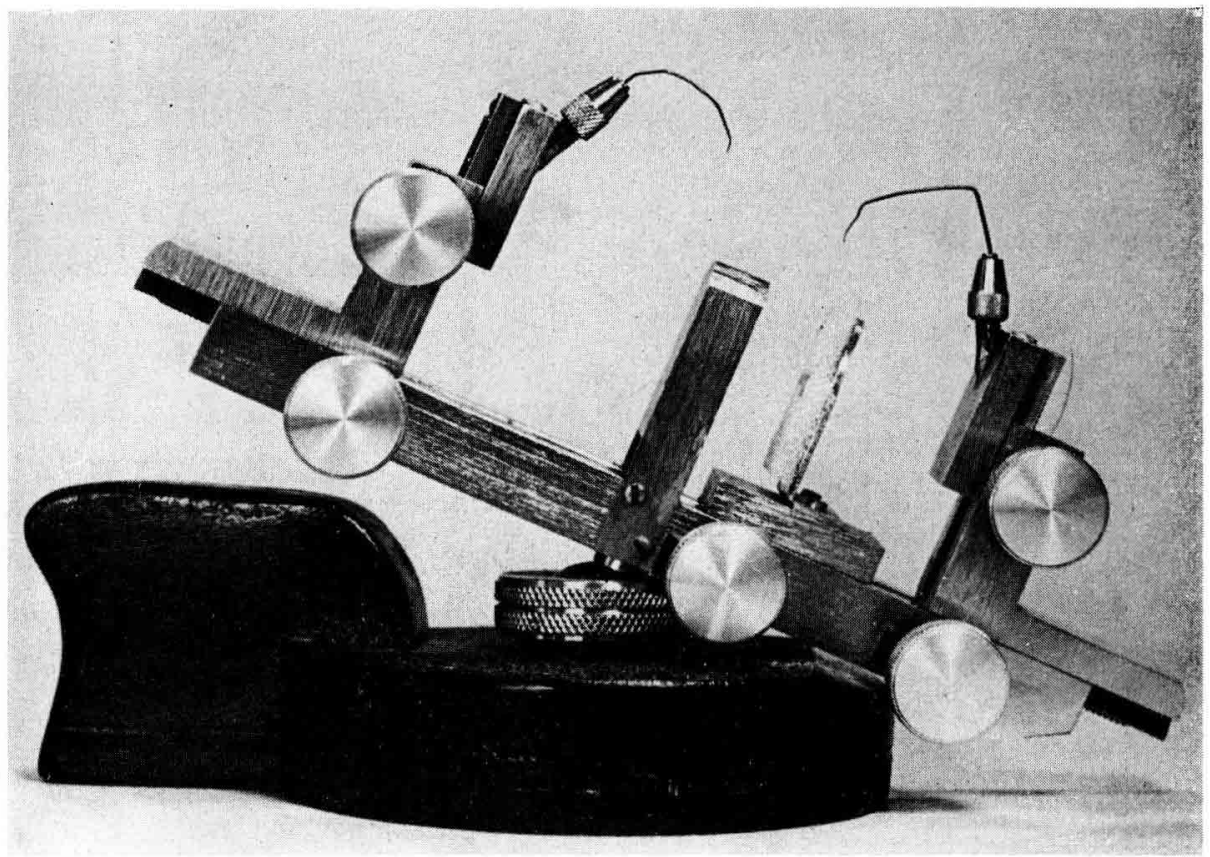

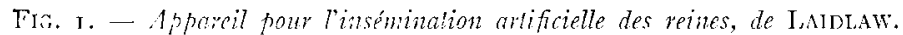

Vue du rité de l'opcrateur montrant les vis micromótriques qui actionment les crochets et l'étau de la chambre de la reine. L'appareil de série a été modifié pur nos besoins: l'inclinaison a été augrmentée, le socle ćpaisici, une poignéa facilite les déplacements

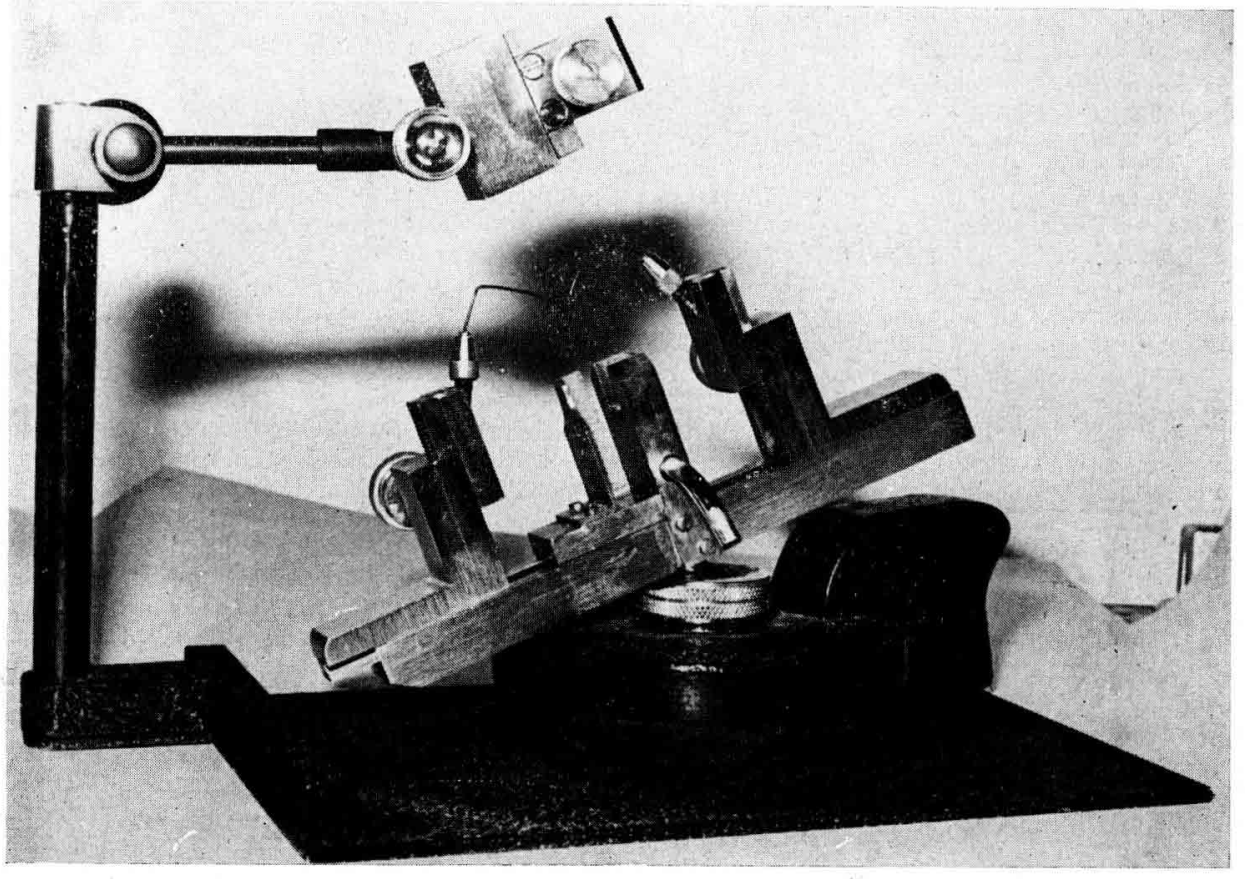

Fig. 2. - Appareil de Laidlaw

Vue du côté opposé à l'opérateur montrant, au centre, le tube d'arrivée du gaz carbonique. L'appareil de contention de la reine est placé ici sur le socle de l'appareil de fixation de la seringue. Une vis micrométrique permet la montée et la descente de celle-ci.

Annales de l'Abeille. - 1966. 
Le crochet dorsal ou crochet de l'aiguillon doit être finement usiné. L'extrémité forme une spatule de $0,77 \mathrm{~mm}$ de diamètre suivie d'un étranglement de $0,08 \mathrm{~mm}$ de large. Sa courbure doit être très étudiée. Le crochet ventral est plus simple; il suffit qu'il ait la courbure convenable et qu'il soit aplati à son extrémité, dans le sens de la largeur, comme la spatule du crochet dorsal. Ces deux crochets, dont nous verrons plus loin l'utilisation peuvent être fabriqués en fil de laiton ou en fil d'acier de $0,7 \mathrm{~mm}$ de diamètre environ. La sonde vaginale est également usinée en laiton ou fil d'acier, elle est amincie à son extrémité et légèrement recourbée.

Le support de la seringue permet la présentation convenable de cette dernière au-dessus de la chambre d'anesthésie de la reine. Un étau permet le blocage de la seringue ; une vis micrométrique assure une descente précise et sans heurt.

La seringue de MACKENSEN est généralement utilisée avec l'appareil de LAIDLAw. Elle est composée d'un tube en acier inoxydable, dans lequel un piston peut être introduit en le vissant et fait ainsi pression sur un opercule en caoutchouc, permettant d'aspirer ou d'injecter le sperme. La pointe de la seringue est en plexiglas. Transparente, elle permet de voir le sperme à l'intérieur. Des graduations indiquent le volume de sperme qu'elle contient.

L'insémination artificielle se pratique sous une loupe binoculaire; nous utilisons habituellement une loupe "Zeiss" à grossissement $\times$ I6.

\section{TECHNIQUE DE L'INSÉMINATION ARTIFICIELLE}

La technique de travail varie légèrement suivant le personnel disponible. Il est difficile d'opérer seul, le prélèvement des reines vierges et des mâles implique un travail et un équipement apicoles ainsi que des déplacements nombreux entre le laboratoire et le rucher. L'insémination exige une asepsie totale, d'où une perte de temps considérable. A partir de deux personnes le travail peut se dérouler facilement et rapidement. Un apiculteur apporte les reines et les mâles en fonction des besoins et rend les reines inséminées à leur colonie dès que l'opération est terminée. Elles sont ainsi un minimum de temps hors de la ruche. L'inséminateur peut alors se consacrer uniquement aux diverses opérations de l'insémination dans les meilleures conditions de rapidité et d'asepsie.

Les divers outils ou appareils qui risquent d'entrer en contact avec les parties génitales des reines, des mâles ou avec le sperme doivent être stérilisés minutieusement. On utilise généralement de l'alcool à $90^{\circ}$ pour les pièces métalliques et un rapide nettoyage à l'alcool à $70^{\circ}$ pour la seringue avec rinçage à l'eau distillée. On peut également employer des antibiotiques. L'infection est l'une des causes majeures de mortalité des reines, lors de l'insémination artificielle, et l'on ne prendra jamais trop de précautions, en ce qui concerne l'asepsie, au cours des diverses manipulations.

Au moment de l'assemblage des diverses parties de la seringue, la cavité qui sert de logement au disque de caoutchouc est remplie d'eau distillée ou de sérum physiologique contenant un antibiotique. La seringue est ensuite fixée sur son support. Une partie du liquide est chassée à l'aide du piston, en vissant celui-ci, de telle façon qu'il soit possible d'amener le liquide jusqu'à l'extrémité de la pointe de la seringue, ou au contraire de le remonter jusqu'au niveau de la cavité contenant le disque de caoutchouc. Il convient de conserver une bulle d'air à l'extrémité de la pointe de la seringue avant de commencer à prélever le sperme afin que celui-ci ne se mélange pas avec le liquide.

Un mâle dont la maturité sexuelle est parfaite est choisi ; on écrase son thorax entre le pouce et l'index pour obtenir le premier stade de l'éversion de l'endophallus. Ce stade peut être obtenu en enfermant les mâles dans un flacon contenant un morceau de coton imbibé de chloroforme, ainsi qu'il est indiqué par MACKENSEN et ROBERTS (I948) ; cette méthode, abandonnée par LAIDLAW (1956) est également déconseillée par Woxke (1965) qui recommande de décapiter les mâles pour obtenir le premier stade d'éversion. Une pression progressive de l'abdomen entre le pouce et l'index permet d'obtenir l'éversion totale du sexe et l'apparition du sperme à l'extrémité du bulbe, à la surface d'une masse de mucus. Le sperme est de couleur beige avec des reflets nacrés, tandis que le mucus est blanc et brillant. La forte contraction des muscles de l'abdomen, sensible au bout des doigts par la rigidité et la résistance à l'écrasement de celui-ci, indique généralement la proximité de l'éjaculation. Les mâles impubères ne présentent pas cette contraction musculaire de l'abdomen et il est vain de tenter d'obtenir du sperme de ceux-ci. Lorsque l'éjaculation s'est produite dans de bonnes conditions le sperme est souvent bien séparé du mucus. Il est alors assez aisé de le prélever à l'aide de la seringue sous la loupe binoculaire, en aspirant lentement à l'aide du piston. Il est très important qu'aucune partie de mucus ne se mélangé au sperme et soit aspirée dans la seringue. Le mucus se coagule rapidement après l'éjaculation et bloque le sperme dans la seringue dont le contenu est alors difficilement récupérable. La présence de mucus lors de l'insémination serait par ailleurs particulièrement néfaste à la reine, car il risquerait de bloquer les oviductes, 
l'entrée de la spermathèque ou du vagin et entraînerait la mort de la reine à plus ou moins brève échéance. Dans la fécondation naturelle le sperme desséché, rejeté par les oviductes et qui n'a pas pu pénétrer dans la spermathèque peut d'ailleurs également causer la mort de la reine (FYG, I963). On s'assure de l'absence de mucus dans la seringue en déplaçant le sperme déjà aspiré, à l'aide du piston, d'avant en arrière et vice versa ; celui-ci doit suivre le mouvement qui lui est imprimé très librement, sans aucune résistance. Il est également très important qu'aucune matière fécale n'entre dans la pointe de la seringue ou vienne en contact avec elle. De même, le sperme ou la seringue ne devront en aucun moment toucher aux poils, au corps, aux pattes, ou à quoi que ce soit qui risquerait de causer une infection, toujours mortelle pour les reines. Suivant le type d'insémination désirée, un ou plusieurs mâles seront utilisés pour une insémination. Il convient de veiller à ce qu'aucune bulle d'air ne se forme entre le sperme des différents mâles utilisés pour une même insémination. Cependant, pendant la préparation des mâles successifs, il est important de reculer temporairement le sperme déjà présent dans la seringue et de créer ainsi une bulle d'air qui évitera le dessèchement qui serait mortel pour les spermatozoïdes.

La reine est anesthésiée au gaz carbonique puis serrée au niveau du thorax dans l'appareil de contention de la chambre d'anesthésie, et placée sous la loupe binoculaire. Une légère admission de gaz carbonique maintient la reine en état d'anesthésie. Le réglage en est facilité par l'interposition, entre la bouteille à pression et l'appareil d'insémination, d'un flacon partieliement rempli d'eau dans lequel plonge le tube d'arrivée du gaz qui arrive alors sous forme de bulles et dont on règle le débit à l'aide d'un détendeur. Un second tube relie le flacon à l'appareil d'insémination. Un coup d'œil de temps à autre sur le flacon permet de surveiller le débit du gaz carbonique, qui doit être lent mais cependant suffisant pour que la reine ne se ranime pas. Pendant que d'une main on maintient la chambre de l'aiguillon de la reine légèrement ouverte à l'aide d'une pince fine, de l'autre et par l'intermédiaire des vis micrométriques correspondantes, on introduit le crochet dorsal dans la cavité de cette chambre et par un mouvement vers la partie dorsale de l'abdomen de la reine on glisse le crochet entre les plaques chitineuses de l'aiguillon, de telle sorte que la spatule du crochet se trouve dessous et que l'étranglement du crochet se trouve entre les plaques. Le crochet et l'aiguillon sont alors l'un près de l'autre. Lorsque la position correcte est obtenue on doit pouvoir baisser ou monter, avancer ou reculer à volonté l'abdomen de la reine à l'aide de ce crochet sans qu'il s'échappe de son logement. Le crochet ventral est ensuite placé de façon à écarter le dernier sternite de

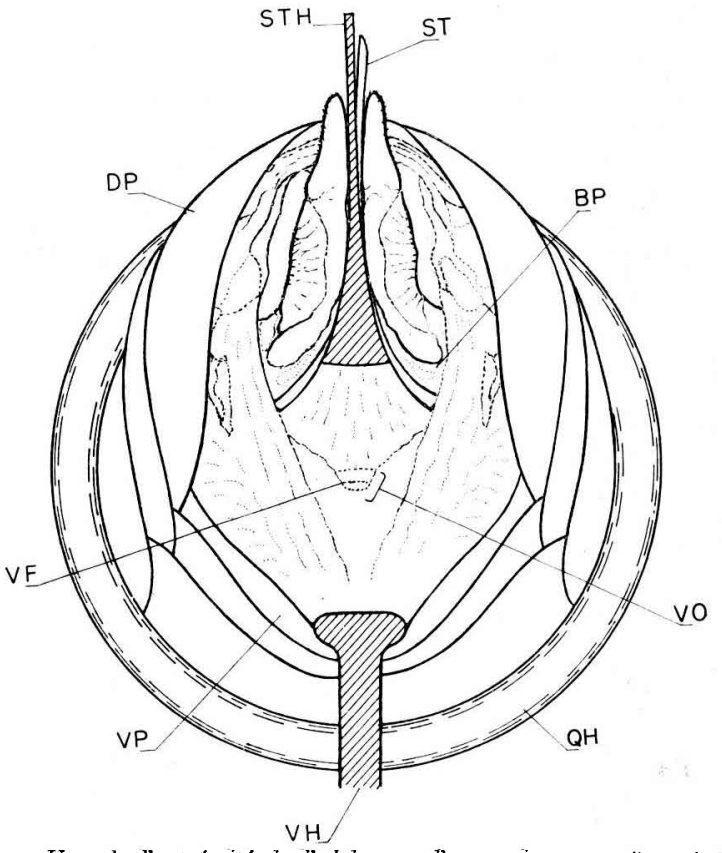

FIG. 3. - Vue de lextrémité de l'abdomen d'une reine correclement préparée pour l'insémination antificielle

STH - crochet dorsal, ST - aiguillon, DP - tergite, VF - repli vaginal, VO - orifice vaginal, VP sternite, $\mathrm{VH}$ - crochet ventral, QH - tube de contention de la reine dans l'appareil de MACKENSEN (D'après Mackensen et Roberts, I948). 
l'abdomen et ouvrir largement la chambre de l'aiguillon. Ia mise en place de ce second crochet est facile ct ne pose aucun problème. Les deux crochets doivent être exactement dans le prolongement l'un de l'autre, faute de quoi l'abdomen de la reine aurait tendance à tourner et à se déplacer ce

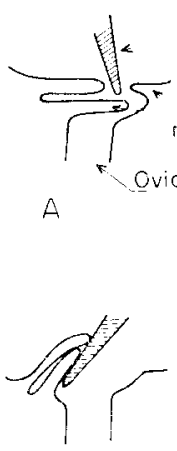

D

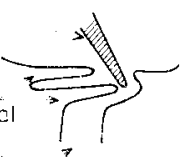

B

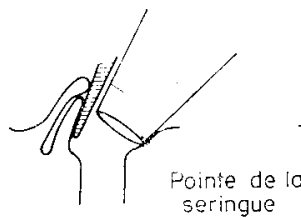

E

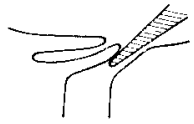

C

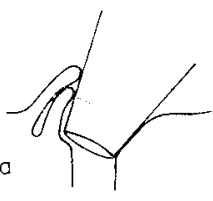

$\mathrm{F}$

Firc. +. - Processus de linsenton de la seringue

(dapres LAIDLAW Ig 56

qui gênerait la suite de l'opération. Thans cutte position (tig. 3) on peut voir, à peu près au centre de la chambre de l'aiguillon le repli vaginal. La seringue est alors ajustée au-dessus de la chambre de l'aiguillon et descendue dans celle-ci de façon à ce cue son extrémité se trouve au-dessus du repli vaginal. La seringue est légèrement remontée; à l'aide d'un coton imbibé d'eau distillée, on hu-

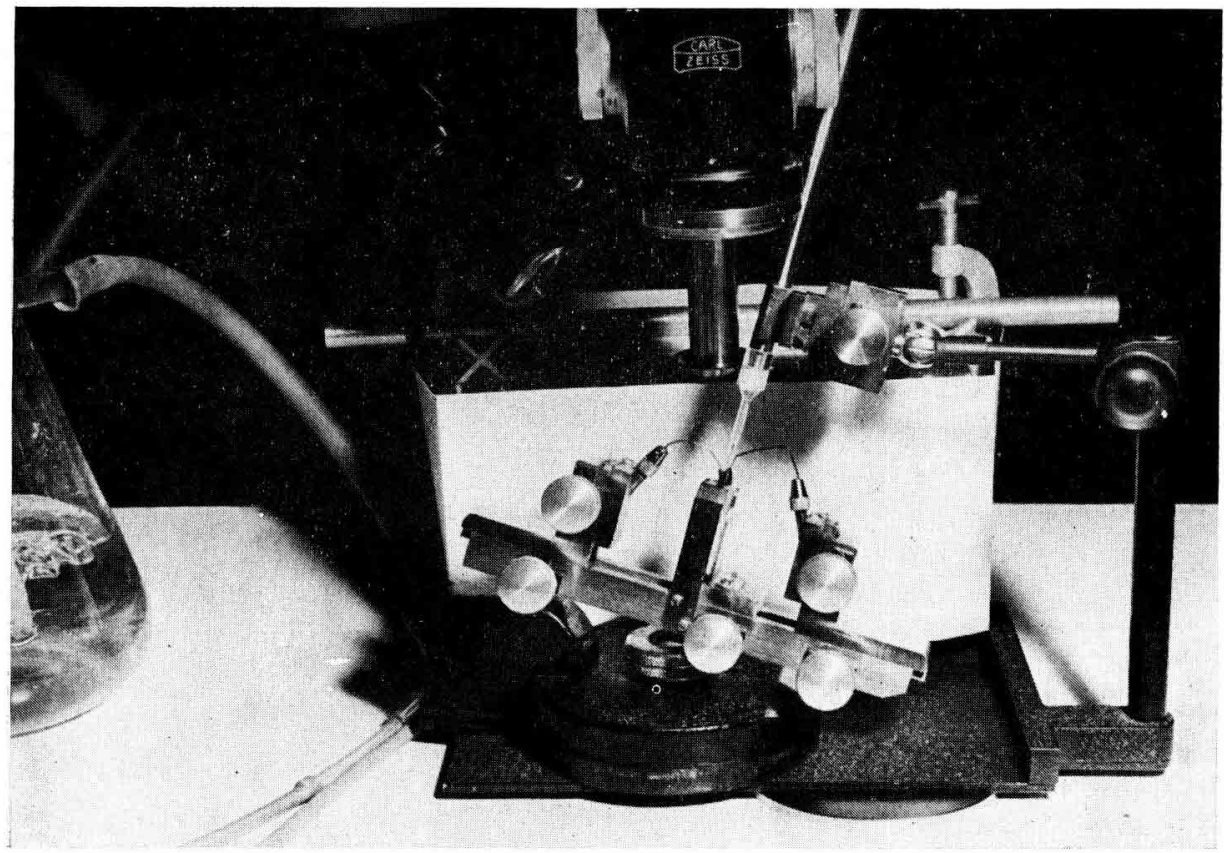

FIG. 5.-- Vue l'ensemble de l'appareil de IAIDLaw pour l'insémination artificielle en position de travail

Appareil de contention de la reine, crochets en position d'écartement des anneaux de l'abdomen, seringue descendue dans l'orifice vaginal. L'ensemble est placé sous une loupe binoculaire à grossissement $\times 16$ 
necte l'extrémité de la pointe ce qui facilite la mise en place. I l'aide de la sonde précédemment décrite, écarter le repli vaginal vers le còté ventral de l'abdomen, puis descendre l'extrénité de la pointe de la seringue dans l'ouverture ainsi ménagée et maintenue par la sonde (fig. 4). ('éle-ci est ensuite enlevée; la seringue doit alors pouvoir légèrement descendre dans le vagim sans blesser la reine et sans enfoncer les tissus environnants, grâce à l'humidification de la pointe. La position cor-

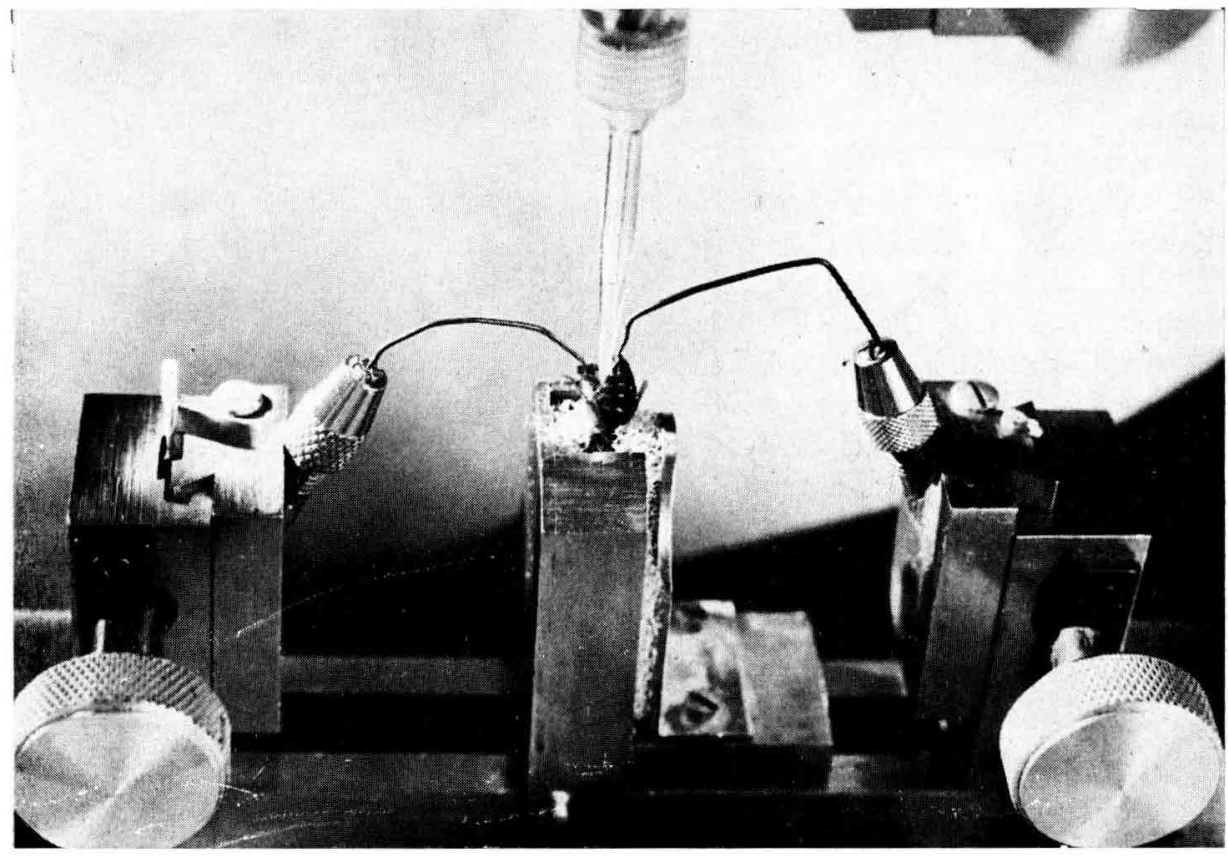

Fis;. 6. - Gros plan de la vue précédente montrant la position des crochets et de la seringue pendant l'insémination d'une reine

recte de la seringue se trouve vérifiée lorsque l'on commence à visser lentement le piston pour injecter le sperme (fig. 5 et 6 ). Si la position est parfaitement correcte le sperme doit descendre facilement sans secousse, et surtout sans remonter autour de la pointe de la seringue. Si le sperme apparaît autour de la seringue il faut cesser immédiatement l'injection, la position de la seringue est défectueuse. Il faut alors remonter celle-ci, réajuster les crochets, puis la seringue, écarter à nouveau le repli vaginal, parfois même déplacer la reine dans la chanbre d'anesthésie. L'abdomen de la reine doit être bien droit, dans le prolongement du thorax et de la tête. Il ne doit pas être renversé dans un sens ou dans l'autre. Il est important d'éviter le télescopage des anneaux ce qui comprimerait les organes. Lorsque le sperme descend normalement, injecter lentement la totalité du contenu de la seringue puis la dégager du conduit vaginal et la remonter. Les crochets peuvent alors être dégagés et éloignés de l'abdomen. La reine est ensuite retirée de la chambre d'anesthésie et placée dans un lieu tempéré en attendant son réveil. Dès (ju'elle est ranimée, elle est introduite dans sa colonie d'origine, généralement un nucleus de fécondation. Une seconde insémination peut être faite de 24 à 48 heures après la première, en suivant le même processus. L'ne grille à reine est placée à la sortie des nuclei afin d'éviter des sorties intempestives des reines. Elles sont en outre marquées et clippées a fin de supprimer tout risque d'envol.

\section{RÉSULTATS}

Le pourcentage de reines qui pondent après avoir été inséminées artificiellement, la qualité et la durée de leur ponte, sont extrêmement variables et tributaires de nombreux facteurs. 
Le début de la ponte a lieu généralement de 3 à 5 jours après l'insémination. Nous avons signalé l'influence déterminante de l'anesthésie au gaz carbonique, qui permet aux reines inséminées de pondre dans un délai presque aussi bref que les reines fécondées naturellement. On considère que deux anesthésies de ro minutes chacune réunissent les conditions les plus favorables à un début de ponte précoce et de qualité normale. Ces deux anesthésies étant déjà appliquées dans le cas de la double insémination d'une reine, il n'y a pas lieu de les renouveler. Cette double insémination est généralement utilisée pour des reines qui doivent pondre longtemps, parfois pendant 3 années.

D'après MACKENSEN et ROBERTS (I948), l'âge le plus favorable à l'insémination artificielle se situe entre le $4^{\mathrm{e}}$ et le Io jours après la naissance. Tous les essais effectués avec des reines plus jeunes n'ont donné que de très faibles pourcentages de réussite. Par contre, les reines peuvent encore être inséminées après ro jours et tant qu'elles n'ont pas commencé à pondre parthénogénétiquement. La ponte parthénogénétique d'une reine n'implique d'ailleurs pas une impossibilité de fécondation artificielle ainsi que l'ont démontré CROW et ROBERTs (I950). Mais à l'âge approximatif de Io jours apparaît le risque, augmenté ensuite tous les jours, de l'attaque de la reine par les ouvrières qui l'obligent à sortir de la ruche pour être fécondée, l'instinct de reproduction n'étant pas inné mais induit par les ouvrières (RUTTNER, I957).

L'âge de fécondation des reines dans les conditions naturelles permet d'estimer 1'âge moyen de la maturité sexuelle et montre sa grande variabilité. SoczEck (I958) signale que les reines volent 6 à I3 jours après leur naissance et MEDVEDEV (I956) indique que les reines fécondées commencent à pondre de 7 à I9 jours après 1'éclosion. Triasko (I95I) utilisant une technique de fécondation naturelle dans de très petits nuclei de fécondation ne laisse sortir les reines que lorsqu'elles ont atteint l'âge de Io à I2 jours.

Nous avons pu démontrer également (FRESNAYE, I966) qu'il existe des variations non négligeables de l'âge auquel les reines atteignent leur parfaite maturité sexuelle, et peuvent donc être fécondées, en fonction de la saison. En prenant pour base une durée d'évolution moyenne de 16 jours entre la ponte de l'œuf et la naissance de l'imago, la maturité sexuelle est atteinte en IO-I2 jours après la naissance au début de la période d'élevage des reines, c'est-à-dire au mois de mars dans notre région. Ce délai diminue progressivement pour atteindre 5 jours après la naissance à partir du mois de juin. Les conditions météorologiques du moment peuvent également faire varier la durée d'évolution des reines.

Le volume du sperme a une très grande influence sur le pourcentage de reines qui survivent et pondent normalement après l'insémination artificielle. WoykE (I963) insémine des reines avec des volumes de I à I6 $\mathrm{mm}^{3}$ de sperme et contrôle la ponte : la mortalité des reines fécondées avec I à $8 \mathrm{~mm}^{3}$ de sperme ne dépasse pas $2 \mathrm{I}$ p. IOO; chez les reines fécondées avec I2 à $\mathrm{I} 6 \mathrm{~mm}^{3}$ elle atteint respectivement 60 et 67 p. Ioo. Il démontre également que parmi les reines inséminées avec I à $4 \mathrm{~mm}^{3}$ beaucoup s'accouplent ensuite naturellement. L'augmentation des doses de sperme abaisse le pourcentage des reines qui sont fécondées naturellement.

Les meilleurs résultats sont obtenus en inséminant les reines deux fois, à I jour d'intervalle, avec $4 \mathrm{~mm}^{3}$ à chaque insémination soit $8 \mathrm{~mm}^{3}$ pour chaque reine. Fn ce qui nous concerne, cette méthode nous permet d'obtenir des reines fécondes dont 
la ponte est normale pendant 2 à 3 ans. Cependant, MACKEnSEN (I964) ayant inséminé des reines avec des volumes de sperme allant de I à $36 \mathrm{~mm}^{3}$ obtient une ponte normale avec une seule insémination de $\mathrm{I} 6 \mathrm{~mm}^{3}$ de sperme. Il considère qu'une insémination de 3 à $4 \mathrm{~mm}^{3}$ suffit pour obtenir une ponte satisfaisante pendant plusieurs mois. IJans ce dernier cas, généralement pour des reines destinées à des études génétiques, des programmes de sélection ou des expériences diverses, il convient de pratiquer une seconde anesthésie, I jour ou 2 après 1'insémination pour que le début de ponte ait lieu dans les délais normaux.

Lors de la fécondation les spermatozoïdes ne pénètrent pas directement dans la spermathèque de la reine mais dans les oviductes. Ce n'est qu'après vingt quatre heures que la totalité des spermatozoïdes a évacué les oviductes, pénétré dans la spermathèque pour une partie, le reste étant rejeté à l'extérieur des organes génitaux. (RutTiner I956). Ce processus explique le délai d'un jour prescrit entre les deux inséminations successives d'une même reine. Une faible partie seulement de la masse de sperme qui peut être acceptée par les oviductes trouve place dans la spermathèque (FYG, I963), et l'augmentation des doses de sperme injectées abaisse le nombre des spermatozoïdes de chaque mâle dans celle-ci (WoykE, I963).

La quantité de sperme que peut fournir un mâle est variable suivant les races et les individus. Bien que les chiffres de I à $3 \mathrm{~mm}^{3}$ aient été avancés, les moyennes de $0,95 \mathrm{~mm}^{3}$ et de $\mathrm{I} \mathrm{mm}^{3}$ données par MACKENSEN et par WoykE sont beaucoup plus proches des volumes que nous obtenons chez les mâles de race noire. Le nombre de spermatozoïdes pour I $\mathrm{mm}^{3}$ de sperme est d'environ 7,64 millions (MACKENSEN, I964).

KOEHI.ER (I962) a démontré que le sperme des différents mâles qui participent aux fécondations, naturelles ou artificielles, reste séparé dans la spermathèque, ce qui explique que parfois on observe de brusques variations biométriques dans certaines populations et l'apparition d'abeilles hybrides dans des ruches considérées comme pures sans que la reine ait été changée.

I a conservation du sperme de faux-bourdon a été réalisée par TABER et BLUM (I960-I96I). Stocké dans des tubes de verre scellés, des fécondations normales ont été obtenues avec ce sperme après 68 jours de stockage. Les températures de conservation les plus satisfaisantes sont comprises entre $20^{\circ}$ et $35^{\circ}$.

\section{MATÉRIEL, ETT MÉTHODES D'ÉLEVAGE}

\section{Les reines}

Il existe de nombreuses méthodes d'élevage des reines. Leur étude a fait 1'objet d'innombrables publications dont 1'analyse sortirait du cadre de notre revue. Il faut cependant souligner les grandes différences de qualité des reines obtenues par les différentes méthodes utilisées actuellement. Par ordre de valeur décroissante elles se répartissent de la façon suivante:

a) L'élevage naturel au moment de l'essaimage, difficilement utilisable dans un élevage moderne en raison du petit nombre de reines que l'on peut obtenir de chaque souche. 
b) L'élevage par transport d'ceuf; (méthode ÖRöSI-PAL, Ig6r-Ig63) encore très peu répandu et de pratique délicate.

c) L'élevage par double greffage. Les larves d'un premier greffage sont remplacées par de nouvelles jeunes larves après I jour d'élevage. Ainsi les futures reines sont déposées sur un lit de gelée royale absolument fraîche, de composition adéquate à l'âge des larves et sans aucune souillure due à des manipulations diverses. Nous avons adopté cette méthode qui donne de très bons résultats.

d) L'élevage ordinaire par greffage de larves jeunes, dérivé de la méthode de production de gelée royale, employé actuellement par la plupart des éleveurs de reines en raison de sa simplicité. Mais les reines obtenues sont de qualité nettement inférieure à celles issues des méthodes précédentes.

Les cellules royales sont placées, peu de temps avant la naissance des reines, dans de petites colonies d'abeilles orphelines, ou nuclei, de taille et de conception très variables. Nous utilisons des hausses "Dadant " divisées en trois compartiments de trois cadres chacun. Les jeunes reines vierges destinées à l'insémination artificielle sont marquées, clippées et claustrées dans les nuclei à l'aide d'une grille à reine qui évite les sorties intempestives.

\section{Les mâles}

L'obtention de mâles nombreux et de souches déterminées est plus difficile et plus aléatoire que l'élevage des reines. Les colonies d'élevage doivent être renforcées au début du printemps par du couvain operculé, des paquets d'abeilles, ou un nourrissement stimulant. Un rayon à cellules de mâles, préalablement construit est ensuite introduit au centre de chaque souche. Si le nombre de mâles nécessaire pour une souche dépasse 400 à 500 , il convient de prélever le cadre après que la reine l'a garni d'œufs, environ 5 jours après l'introduction, et de le placer dans une colonie qui assurera l'élevage. Un nouveau cadre vide remplacera le premier et sera à nouveau rempli d'oufs par la reine.

BANBy et LAIDLAW (I96I) conseillent de faire élever les mâles dans de fortes colonies sans reine car les colonies normales risquent de détruire le couvain de mâles qui leur est donné à nourrir.

Des reines vierges traitées au gaz carbonique sont un moyen sûr pour obtenir des mâles (Mackensen et Roberts, I948). Deux jours avant la date de naissance présumée les rayons sont retirés des colonies éleveuses, introduits dans des boîtes à cadres et placés à l'étuve à $34^{\circ}$. Le jour de la naissance ou le jour suivant les mâles sont introduits dans des cages de maturation, dont une face est garnie de grille à reine, et placés dans une colonie nourrice. De même que pour l'élevage, les colonies nourrices sans reine sont préférables, car les colonies normales laissent fréquemment les mâles encagés dans la ruche mourir de faim. Ils sont pubères 8 à io jours plus tard et survivent bien dans ces conditions pendant encore Io à I 5 jours (LAIDLAW I956). Mais d'après KuRENOI (I953) quelques mâles seulement sont mûrs à I2 jours ; la plupart n'atteignent leur parfaite maturité sexuelle qu'à 20 jours. Comme pour les reines et les ouvrières il y a des différences raciales de la durée d'évolution. L'élevage des mâles doit donc débuter 45 jours au moins avant la date de l'insémination artificielle.

D'après JAyCox (I96I) les mâles encagés sans ouvrières à $34^{\circ}$, nourris au miel 
et at pollen, parfois avec addition de gelée royale, atteignent leur maturité aussi bien que lorsqu'ils sont encagés dans les colonies nourrices ou en vol libre. A 3 I $^{\circ}$ elle serait légèrement retardée et à $28^{\circ}$ très retardée. Cependant, MACKENSEN (I954) recommande de laisser voler librement les mâles car l'insémination avec des mâles confinés est 1'une des causes majeures de la mort de beaucoup de reines. Il obtient 82 p. Ioo de reines survivantes inséminées avec le sperme de mâles ayant volé librement et seulement $44 \mathrm{p}$. Ioo chez celles inséminées avec le sperme de vieux mâles confinés. Par ailleurs Woyke (I955) a mis en évidence l'action du vol sur l'excitabilité des faux-bourdons. Il obtient 4 fois plus d'éjaculations complètes chez des mâles rentrant d'un vol que chez ceux n'ayant pas volé. Or, la dérive des mâles est très importante dans les conditions naturelles de vie des colonies. GoETZE (I954) a trouvé que dans certaines colonies $50 \mathrm{p}$. Ioo des mâles étaient d'origine étrangère. Afin de connaître avec certitude les faux-bourdons utilisés en insémination artificielle il est donc indispensable de supprimer cette dérive.

Pour pallier ces divers inconvénients nous avons remplacé l'encagement des mâles dans les ruches par l'emploi de cages spécialement conçtres, placées autour des ruches à mâles (FRESNAyE, I 964). Les mâles volent et s'orientent très bien dans ces cages et atteignent une parfaite maturité. Les abeilles peuvent sortir à l'extérieur et approvisionner les colonies sans perturbation.

\section{CONCLUSION}

L'insémination artificielle des reines d'abeilles a bénéficié d'améliorations importantes depuis la création de l'insémination instrumentale. Nous avons fait une revue succincte des progrès les plus déterminants.

En conclusion nous résumerons les facteurs les plus importants qu'il convient de réunir pour obtenir un maximum de chances de succès dans l'insémination artificielle des reines.

I ${ }^{0}$ Asepsie. - Une asepsie parfaite est la condition primordiale et impérative du succès dans l'insémination artificielle. Un très fort pourcentage de la mortalité enregistrée chez les reines inséminées est đû̀ à des infections diverses (infection manuelle, par les outils ou les appareils, par contact avec le corps des mâles, des reines ou de matières fécales).

$2^{\circ}$ Dextérité. - L'insémination artificielle des reines ne demande pas une habileté manuelle particulière, il convient cependant de pratiquer beaucoup afin d'opérer rapidement, sans hésitation et d'éviter de blesser les reines.

$3^{\circ}$ Le volume de sperme injecté lors de chaque insémination ne doit pas être trop important; les meilleurs résultats sont obtenus en pratiquant deux inséminations à 24 heures d'intervalle et en utilisant $4 \mathrm{~mm}^{3}$ de sperme à chaque dose.

$4^{\circ}$ Aucune parcelle de mucus ne doit pénétrer dans la seringue lors du prélèvement du sperme sur le faux-bourdon et il ne doit pas en subsister sur les parois extérieures de celle-ci. En se coagulant le mucus bloque 1'entrée des oviductes, du vagin ou de la spermathèque. 
$5^{\circ}$ L'insémination doit être pratiquée sur des reines dont la maturité sexuelle est complète. Il convient de varier les délais entre la naissance et l'insémination en fonction de la saison et des conditions météorologiques du moment.

$6^{\circ}$ Les mâles doivent avoir atteint leur parfaite maturité. Celle-ci est obtenue entre le $\mathrm{I} 2^{\mathrm{e}}$ et le $2 \mathrm{O}^{\mathrm{e}}$ jour après la naissance. Il convient également d'éviter d'utiliser les mâles très âgés.

$7^{\circ}$ Il est préférable de provoquer l'éjaculation des mâles par décapitation plutôt qu'en les anesthésiant au chloroforme.

$8^{\circ}$ Les mâles doivent avoir volé avant leur utilisation.

Le respect de toutes ces règles doit permettre d'obtenir un pourcentage de succès proche des roo p. Ioo. Cependant il faut considérer que même dans les conditions naturelles de fécondation il arrive très fréquemment que l'on n'obtienne pas une réussite totale. En dehors des facteurs extérieurs, pertes de reines par dérive ou mangées par les oiseaux, mauvais temps, il se produit également une certaine mortalité due à des maladies infectieuses (FyG, I934) ou a un blocage de sperme dans les organes génitaux des reines. Ces conditions peuvent se retrouver dans l'insémination artificielle sans qu'il y ait eu faute de la part de l'opérateur.

$$
\text { Reçu pour publication en juin } 1966 .
$$

\title{
SUMMARY
}

\author{
THE ARTIFICIAL INSEMINATION OF QUEEN BEES
}

A concise review is given of the most important works on recent progress in artificial instrumental insemination of queen bees and a detailed description is given of the method used.

\section{RÉFÉRENCES BIBLIOGRAPHIQUES}

Banby H. H., Laidlaw W. C., ig6r. Drone production. Bee Genetics, 2, I9.

Chauvin R., i95o. Sur l'insémination artificielle des reines d'abeilles sans appareils compliqués. Apiculteur (Sect. Sci.), 5, 69-74.

Crow J. F., Roberts W. C., I950. Inbreeding and homozygosis in bees. Genelics, 35, 61 2-621.

FrESNAYE J., I 964 . La claustration des mâles destinés à l'insémination artificielle des reines d'abeilles. Ann. Abeille, 7( (1), 55-61.

FRESNAYE J., I 966 . Infuence des variations de l'âge de maturité sexuelle chez les reines d'abeilles (Apis mellifica mellifica) fécondées par insémination artificielle. Ann. Abeille, 9 (3), 237-242.

FYG W., I934. Krankheiten der Bienenkönigin. Schweiz. Bienenztg, 57, 458-467, 504-514, $570-571$.

Fyg W., 1963. Anomalies et maladies des reines d'abeilles. Bull. Apic. Inform., 6, (1), 37-151.

Goetze G., I954. Wie stark verfliegen sich die Drohnen an einem grosser Bienenstand. Leipzig. Bienenzgt. (West.), 63 (9), 223-225.

J $\Lambda$ Ycox E. R., ig6r. The effects of various foods and temperatures on sexual maturity of the drone honey-bee. Ann. Entomol. Soc. Amer., 54, (4), 519-523.

KoeHLer F., I962. Recherches expérimentales sur la fertilité du receplaculum seminis à l'aide de la fécondation artificielle. Bull. Apic. Inform., (2), 2 19-224.

Kurenor N. M., r953. Quand les mâles sont-ils sexuellement mûrs? (en russe). Pchelovodstro, 11, $28-32$.

Lafdlaw H. H., I932. Hand mating of queenbees. Amer. Bee J., 72 ( 7 ), 286.

LAIDLAW H. II., I944. Artificial insemination of the queen-bees (Apis mellifica L.). Morphological basis and results. J. Morphology, $74(3), 429-465$. 
LAIDLAW H. H., I949. Development of precision instruments for artificial insemination of queen-bees, J.econ. Entomol., 42 (2), 254-26I.

LAIDLAW H. H., I949. New instruments for artificial insemination of queen-bees. Amer. Bee J., 89 (I 2). $566-567$.

LAIDLAW H. H., I953. An anesthetization chamber for the artificial insemination of queen-bees. J. econ. Entomol, 46 (I), 167 .

Laiblaw H. H., I956. Organization and operation of a bee breeding program. Proc. Xih Intern. Congr. Enionol., 4, I $067-1078$.

Mackensey O., 1947. Effect of carbon dioxide on initial oviposition of artificially insemination and virgin queen-bees. j. econ. Entomol. 40 (3), 344-349.

Mackensen O., Roberts W. C., I948. A manual for the artificial insemination of queen-bees. U.S.D.A. Bur. Ent. and Plant. Quar. E. T., 250, 33 pages.

Mackensen 0 , 1948. A new syringe for the artificial insemination of queen-bees. Amer. Bee $J ., 88$ (8), $4 \mathrm{I} 2$.

Mackensen $0 ., 1954$. Some improvements in method and syringe design in artificial insemination. I. econ. Entomol, $48(5), 765-768$.

Mackensen O., 1955. Experiments in the technique of artificial insemination of queen bees. J. econ. Enlomol., 48 (4), +18-421.

Mackensen O., I964. Relation of semen volume to success in artificial insemination of queen honey-bees. J.econ. Enionol., 57 (4), $58 \mathrm{I}-583$.

Medvenev I. K., I956. Pchelovodstvo (33), ro-13.

Nol.an W. J., I932. Breeding of the honey-bee under controlled conditions . U.S.D.A. Tech. Bull. $3^{26}$.

Notan W. J., 1937. Improved apparatus for inseminating queen-bees by the Watson method. $J$. econ. Entomol., 30, 700-705.

ÖRösI-PAL, I96r. Nouvelles expériences, sur l'élevage de reines à partir de l'œuf. Meheszet, Budapest, $9(8), \mathbf{1} 47^{-1} 48$.

ÖÖSI-PAL, I963. Résultats de l'élevare de reines à partir de l'œuf. Mehesэét, Budapest, 11 (8), I43-I45.

Rutrner F., 1956. Zur frage der Spermaübertragung bei der Bienenkönigin. Insectes Sociaux, 3 (2), 35 1359 .

RutTiner F.. i95\%. Die Sexualfunktion der Honigbienen im Dienste ihrer sozialen Gemeinschaft. $Z$. vergleich. Phy'siol, 39, 577-600.

Rutrner F.. r964. Zur Technik und Anwendung der Kunstlichen Besamung der Bienenkonigin. Z. Bienenforsch., 7 (2), $25-34$.

Suith M. V., I96ı. A note on natural mating under artificial conditions. Bee World, 42 (7), 182.

Soczeck Z., 1958. Influence of some factors on queens'flights and matings. Pszc. Zesz. Naukoree 2, rogII9.

Taber S., Blem V. S., ig60. P'reservation of honey bee semen. Science, 131 (34I5), $1734^{-1} 735$.

TaBer S., I961. Successful shipments of honeybee semen. Bee world, 42, (7), 1 $73^{-1} 76$.

Triasko V. V., I95I. Méthode de fécondation des reines dans de petits nuclei. Pchelovodstvo, (9), I8-25.

TrRIASKo V. V., I958. Une appréciation de la méthode manuelle d'insémination artificielle des reines. Byull. Inst. Pchelovodstva, $(\mathbf{3} / \mathbf{4}), 5^{-8}$.

VESELy V., I96I. Sur le problème de l'insémination artificielle des reines d'abeilles (en tchèque). Fol. Zool., $10(3), 203^{-210 .}$

Watson L. R., 1927. Controlled mating of queen-bees. Hamilton-Ill.

Woyke J., r955. Multiple mating of the honeybee-queen in one nuptial flight. Bull. Acad. Polon. Sci., 11, $3(5)$, I $75^{-1} 80$.

Woyke J., r963. L'action de plusieurs faux-bourdons sur l'insémination de la mère. XIXe Congrès Apimondia, 2, 763-766.

Worke J., 1963. Le comportement des reines fécondées artificiellement de diverses façons. XIXe Congrès Apimondia, 2, 767-768.

Woyke J., 1965. (Communication verbale.) 